\title{
A Computer-Based Technique for Automated Spot Detection in Proteomics Images
}

\author{
Eleftheria A. Mylona, Michalis A. Savelonas, Member, IEEE, Dimitris Maroulis, Member, IEEE, and Sophia Kossida
}

\begin{abstract}
This paper introduces a novel computer-based technique for automated detection of protein spots in proteomics images. The proposed technique is based on the localization of regional intensity maxima associated with protein spots and is formulated so as to ignore rectangular-shaped streaks, minimize the detection of false negatives, and allow the detection of multiple overlapping spots. Regional intensity constraints are imposed on the localized maxima in order to cope with the presence of noise and artifacts. The experimental evaluation of the proposed technique on real proteomics images demonstrates that it: 1) achieves a predictive value $(P V)$ and detection sensitivity $(D S)$ which exceed $90 \% ; 2$ ) outperforms Melanie software package in terms of $P V$, specificity, and $D S ; 3$ ) ignores artifacts; 4) distinguishes multiple overlapping spots; 5) locates spots within streaks; and 6) is automated and efficient.
\end{abstract}

Index Terms-Proteomics images, protein spot detection, regional extrema.

\section{INTRODUCTION}

$\mathbf{T}$ WO-DIMENSIONAL polyacrylamide gel electrophoresis (2-D PAGE) [1] is a powerful technique in proteomics aiming at protein separation and identification. 2-D PAGE is performed using isoelectric focusing and sodium dodecyl sulfate polyacrylamide gel electrophoresis (SDS PAGE), which allow protein separation in terms of their charge and molecular weight, respectively. Each resulting gel contains a few hundred up to several thousands of protein spots. The aim of gel analysis is to seek for alterations in protein expression, which correlate with pathological conditions, such as cancer. Accordingly, it is vital to detect protein spots that have appeared or disappeared during the various cell-cycle stages.

2-D PAGE imaging poses challenges in current image analysis research, considering that gel images feature media structure differences, variations in the electric field applied, as well as the presence of noise, dust particles, fingerprints, and cracks on

Manuscript received September 23, 2010; revised February 8, 2011; accepted March 26, 2011. Date of publication April 7, 2011; date of current version July 15, 2011. This work was supported in part by the European Union (European Social Fund) and Greek National Funds through the Operational Program "Education and Lifelong Learning" of the National Strategic Reference Framework-Research Funding Program: Heracleitus II. Investing in knowledge society through the European Social Fund.

E. A. Mylona, M. A. Savelonas, and D. Maroulis are with Realtime Systems and Image Analysis Group, Department of Informatics and Telecommunications, University of Athens, 15784 Athens, Greece (e-mail: emylona@ di.uoa.gr; m.savelonas@di.uoa.gr; d.maroulis@di.uoa.gr).

S. Kossida is with the Biomedical Research Foundation of the Academy of Athens, 11527 Athens, Greece (e-mail: skossida@bioacademy.gr).

Digital Object Identifier 10.1109/TITB.2011.2140327 the gel surface. In addition, difficulties arise due to the nonuniformity of the background intensity and the large variations of protein expression, which range from faint to saturated spots. Another open issue comes from the complex regions containing multiple overlapping spots. Such "multiplets" tend to occupy a large portion of the gel surface and their detection is often very difficult. Currently, biologists perform a laborious, errorprone process involving the correction of the output generated by 2-D PAGE image analysis software packages, such as Melanie (Geneva Bioinformatics SA, Geneva, Switzerland) [2]. The development of automated and consistent computer-based spot detection techniques is of crucial importance.

State-of-the-art approaches to spot detection include watersheds [3] and stepwise thresholding [4]. Nonetheless, watersheds lead to the detection of false positives [5], whereas stepwise thresholding fail in the presence of artifacts and noise [6]. The more recently proposed watershed-based approach of Tsai et al. [7] fails in the presence of multiplets.

Mathematical morphology [8] is a well-known image analysis approach. It can be applied for the extraction or suppression of image components of interest by designing a suitable structuring element (SE) [9]. It is based on set theory, considering images as sets of points. Set operations such as union, intersection, addition, and subtraction are performed between two sets: the object of interest and the SE. The key morphological operations are dilation and erosion, forming the basis for more complex operations such as opening and closing. Morphological operations are capable of preserving topological properties such as connectivity and homotopy, whereas they are suitable for detecting the location of geometric characteristics, such as intensity peaks [10]-[14]. They have been successfully applied on various biomedical domains, including electron micrographs of muscle cross sections [15], gastric tumor pathologic cell images [16], digital color eye fundus images [17] as well as on 2-D PAGE imaging [18], [19].

This paper introduces a novel computer-based technique for automated detection of protein spots in 2-D PAGE images. Emerging from the association of regional intensity maxima with protein spots, the proposed technique utilizes the dilation image operator. The dilation operator embeds a disk-shaped SE, adjusted to the dominant roundish shape of protein spots. The disk-shaped SE limits the falsely detected streaks. SE size is set considering that a certain radius value minimizes the detection of false negatives $(F N)$, whereas it allows the detection of local maxima associated with small spots even in cases where they overlap with larger spots in complex regions. The proposed technique imposes regional intensity constraints on the dilationgenerated maxima so as to cope with the presence of noise and 

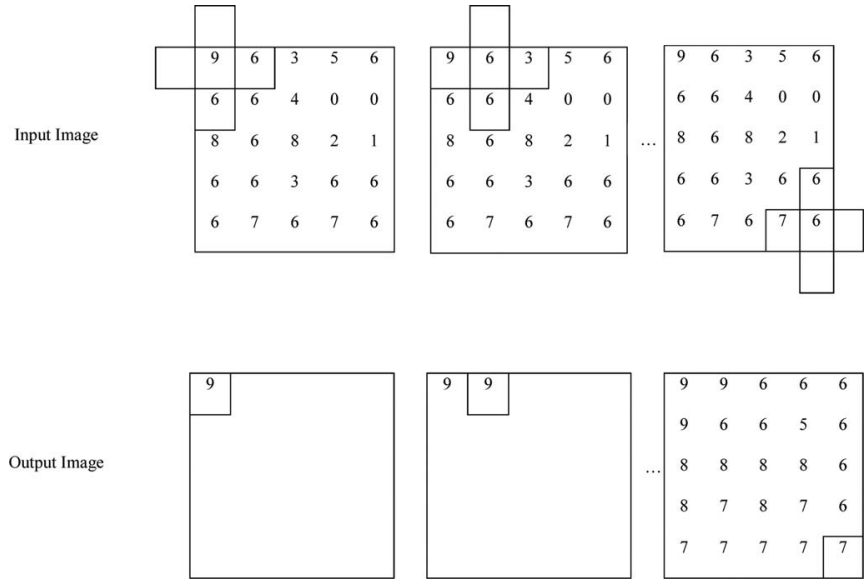

Fig. 1. Dilation operator with a 4NN SE on a sample image.

artifacts. Furthermore, it is automated, efficient, and consistent with the time-consuming work of biologists [20], [21]. As such, it extends the preliminary 2-D PAGE image analysis approach proposed in [22].

The outline of this paper is organized in five sections. Section II presents an overview of mathematical morphology, whereas Section III presents the main components of the proposed technique. Section IV demonstrates the experimental results on real 2-D PAGE images, as well as comparisons with Melanie 7 (Geneva Bioinformatics SA, Geneva, Switzerland), a renowned 2-D PAGE image analysis software package. Finally, conclusions and future perspectives of this paper are discussed in Section V.

\section{Overview of Mathematical MorPhOlOGY}

\section{A. Background}

Dilation and erosion are the two key morphological operations [23]. Dilation involves the intensity transformation of each pixel $A(x, y)$ to the maximum intensity of a region centered on $(x, y)$. Erosion is similar, differing only in the sense that it transforms to the regional intensity minima. Each region is specified by an SE defined by $B(i, j)$ over a domain $B_{\Delta}$. Pixel values within the region of the SE are either 1 or 0 . The morphological operation is applied on the neighborhood formed by pixels of value equal to 1 . The gray-scale dilation operator of $A(x, y)$ with the SE $B(i, j)$ is denoted as $A \oplus B$ and defined by

$$
A \oplus B=\max \left\{A(x+i, y+j)+B(i, j) \mid(i, j) \in B_{\Delta}\right\} .
$$

The gray-scale erosion image operator of $A(x, y)$ by the SE with the function $B(i, j)$ is denoted by $A \Theta B$ and defined by

$$
A \Theta B=\min \left\{A(x+i, y+j)-B(i, j) \mid(i, j) \in B_{\Delta}\right\} .
$$

Fig. 1 depicts the dilation image operator on a sample image with a four nearest neighbor (4NN) SE. The SE is placed over the upper left pixel of the input image and slides along to the next pixel until the whole image has been processed. The maximum intensity value within the SE $B(i, j)$ centered to each $A(x, y)$ is set to the $(x, y)$ pixel of the output image. The erosion image operator works in a similar fashion.
Variations in SE size and shape may alter the effect of morphological operations and should be taken into consideration [24]. The shape of the SE is often selected in accordance with the shape of the objects of interest. Square and rectangular shapes are selected when the focus is put on edge detection, whereas in some cases a disk-shaped SE unrelated to directions may lead to better results. On the other hand, the size of the SE is selected in accordance with the size of the image components. For instance, a small disk tends to detect a large amount of regional intensity maxima, whereas a large disk tends to ignore most of such maxima.

\section{B. Regional Intensity Extrema}

Regional intensity maxima and minima are vital morphological image features, usually associated with bright or dark objects of interest. A regional maximum $M$ at elevation $t$ is defined by

$$
\begin{cases}I(p)=t, & \forall p \in M \\ I(p)<t, & \forall p \in \delta_{\mathrm{SE}}(M) \backslash M\end{cases}
$$

where $p$ is the pixel location, $I(p)$ is the intensity of $p$, and $\delta_{\mathrm{SE}}$ is the region generated by the dilation of $M$ according to the SE. From (3), it is evident that a regional maximum is not restricted to one pixel but is extended to a connected neighborhood $M$. The main attribute of $M$ is that no higher intensity value exists in $\delta_{\mathrm{SE}}$.

A regional minimum $m$ at elevation $t$ is similarly defined by

$$
\begin{cases}I(p)=t, \quad \forall p \in m \\ I(p)>t, \quad \forall p \in \delta_{\mathrm{SE}}(m) \backslash m\end{cases}
$$

The regional extrema of an image are the union of its regional maxima and minima.

\section{Morphology-BASed Protein Spot Detection}

A morphology-based technique is introduced for the detection of protein spots in 2-D PAGE images. As a first step, a $3 \times 3$ median filter is applied on the 2-D PAGE image, so as to tackle with the presence of noise. The application of the median filter on 2-D PAGE images has been supported in previous works such as [25].

In cases of 2-D PAGE images depicting protein spots as bright regions over a dark background, as the images illustrated in Fig. 2(a), protein spots can be associated with regional intensity maxima. These maxima can be determined by the application of (3).

The SE selected to form the connected regions associated with the regional intensity maxima is disk shaped, approximating the dominant shape of protein spots in these images. SE radius $r$ is set according to the results of preliminary experimentation on 2-D PAGE images, considering that a certain radius value minimizes the detection of $F N$, whereas it allows the detection of local maxima associated with small spots even in cases where they overlap with larger spots in complex regions. Fig. 2(b) illustrates the result of the dilation of the 2-D PAGE image of Fig. 2(a) with the utilized SE. The latter is illustrated in Fig. 3.

A regional intensity maxima selection stage follows dilation. The selection is based on two constraints. 


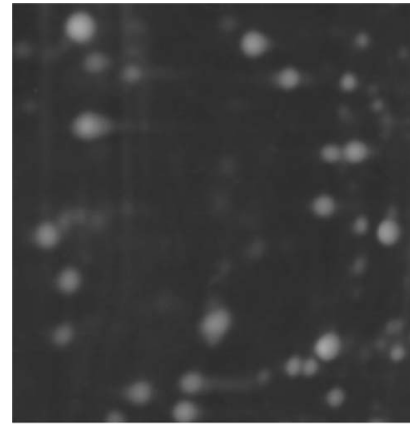

(a)

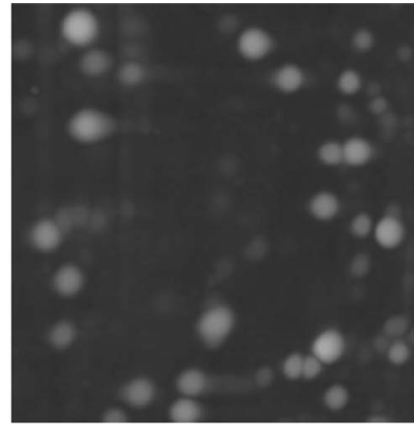

(b)
Fig. 2. (a) 2-D PAGE image containing bright spots over a dark background. (b) Dilation of the image of (a) with a disk-shaped SE.

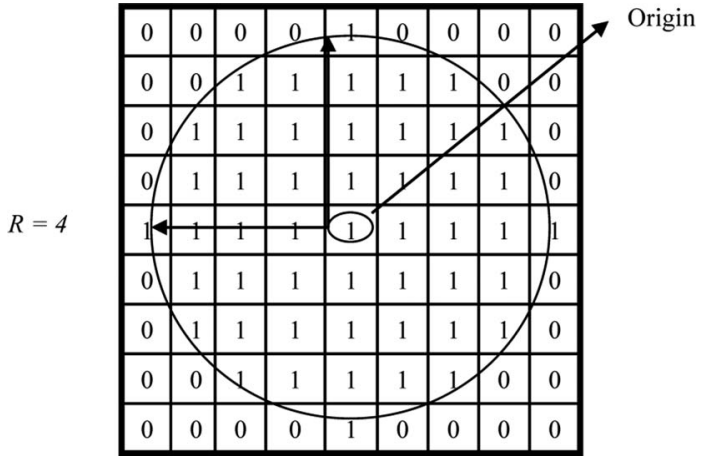

Fig. 3. Disk-shaped SE of radius 4.

1) The intensity of the selected regional maxima should be equal to or higher than a $k \times k$ adjacent region. This constraint is applied so as to include salient maxima associated with spots and avoid extremely local maxima associated with noise.

2) Intensities of the $3 \times 3$ neighborhood of each pixel of the selected regional maxima should exceed a threshold value $T$ in order to avoid the detection of spurious maxima associated with unwanted artifacts and background peaks.

The algorithm of the proposed technique is summarized in the block diagram of Fig. 4.

\section{RESULTS}

The proposed technique has been experimentally evaluated on 13 real 2-D PAGE images provided by the Biomedical Research Foundation of the Academy of Athens. The utilized dataset contains approximately 26000 protein spots. The 2-D PAGE images were obtained in a digital format at 8-bit gray-level depth, with field of view of approximately $56810 \mathrm{~mm}^{2}$ and size of $700 \times$ 650 pixels, whereas the algorithm has been implemented in MATLAB R2009b and executed on a 3.2 GHz Intel Pentium workstation. Three ground truth images were provided by the expert biologists of the Biomedical Research Foundation of the Academy of Athens for each 2-D PAGE image. The replicates for each ground truth image were five. The application of the majority rule on these triads of ground truth images resulted in the final ground truth images used in the experiments performed.

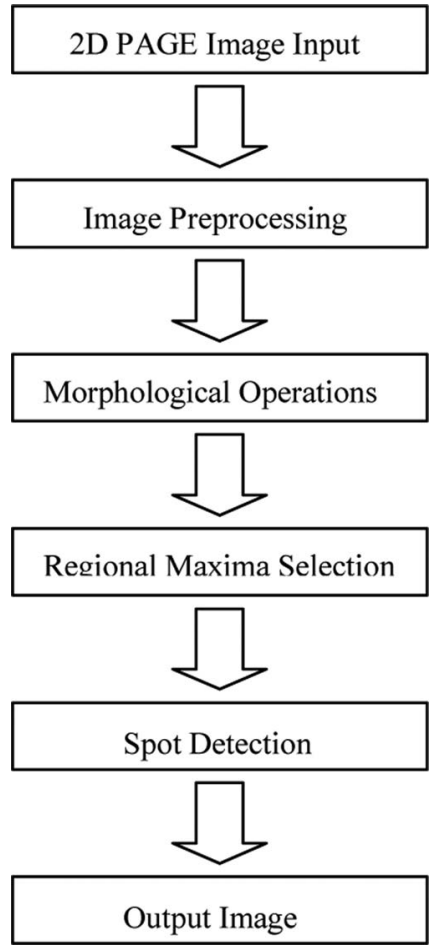

Fig. 4. Block diagram of the proposed technique.

Parameters $T, k$, and $r$ were set to 75,8 , and 4 , respectively, based on the following considerations:

1) threshold value $T$ is selected equal to or lower than the intensity of the faintest spot so as to allow the detection of local maxima associated with faint spots;

2) $k \times k$ size of adjacent region should be equal to or higher than 20 pixels considering that the typical size of a protein spot ranges from 20 to 300 pixels in order to allow the detection of local maxima associated with spots and avoid local maxima associated with noise; and

3) radius $r$ is selected smaller than the smallest protein spot radius in order to allow the detection of local maxima associated with small spots even in cases where they overlap with larger spots in complex regions.

These parameter values were used in all experiments to follow.

Parameters $T, k$, and $r$ are not directly affected by the total image size. However, as it can be derived from the earlier considerations, parameters $k$ and $r$ are affected by the range of protein spot sizes in pixels, which is determined by image resolution. In addition, parameters $k$ and $r$ are invariable to image contrast, as opposed to parameter $T$. The latter decreases as image contrast increases, since contrast increase lowers faint spot intensities, which provide the upper bound of $T$, as described.

The Melanie software package is used for comparisons. Melanie parameters "smooth," "saliency," and "min area" were set to 4,70 , and 60 , respectively. These values were selected by an expert biologist, following his experience. According to the documentation provided with Melanie: 1) "smooth" parameter determines the number of iterative executions of the diffusion 


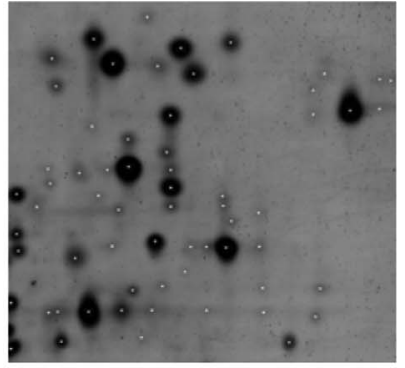

(a)

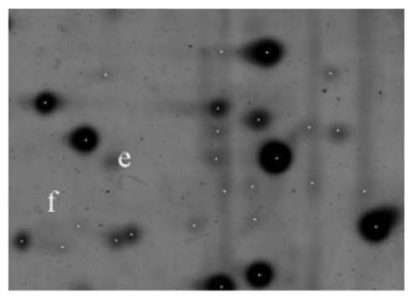

(b)

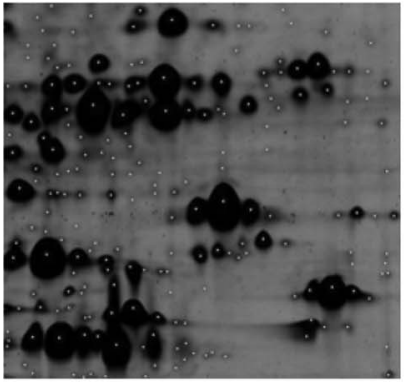

(c)

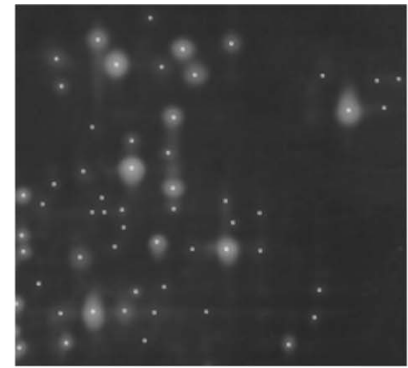

(d)

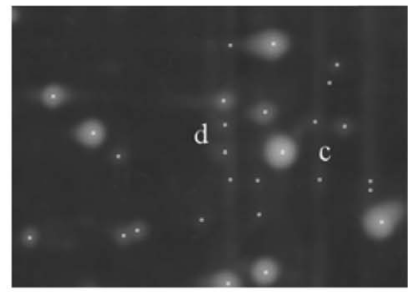

(e)

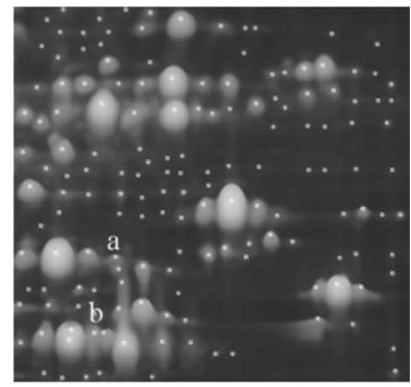

(f)
Fig. 5. (a)-(c) Ground truth images. (d)-(f) Detection results obtained by the proposed technique.

smoothing algorithm, which is a component of Melanie; 2) "saliency" parameter indicates how far a spot stands out with respect to its environment. Real spots generally have high saliency values, whereas artifacts and background noise have small saliencies; 3) "min area" parameter eliminates spots of area smaller than the specified value, which is expressed in number of pixels.

Fig. 5(a)-(c) illustrates three of the final ground truth images, whereas Fig. 5(d)-(f) depicts the detection results obtained by the proposed technique. It is evident that the vast majority of the actual protein spots are correctly detected. The detection results were quantified by means of the predictive value $(P V)$, specificity $(S P)$, and detection sensitivity $(D S)$ [26], which are defined as

$P V=\frac{T P}{T P+F P}, \quad S P=\frac{T N}{T N+F P}, \quad D S=\frac{T P}{F N+T P}$

where true positives $(T P)$ and $F N$ are defined as correctly detected and falsely detected spots. $T P$ and $F N$ are calculated based on the final ground truth images. Table I presents the calculated $P V, S P$, and $D S$ for each 2-D PAGE image in Fig. 5. The proposed technique obtains a $D S$, which exceeds $90 \%$ in all image cases. In the cases of Fig. 5(d) and (e), it misses only four and
TABLE I

IndicAtive Detection Results OBtained By the Proposed TeChNiQuE

\begin{tabular}{cccc}
\hline Image of Fig. 5 & $P V(\%)$ & $S P(\%)$ & $D S(\%)$ \\
\hline d & 92 & 83 & 93 \\
e & 91 & 81 & 92 \\
f & 85 & 77 & 90 \\
\hline
\end{tabular}

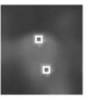

(a)

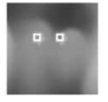

(b)

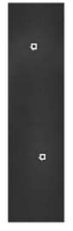

(c)

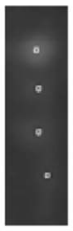

(d)

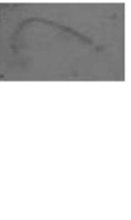

(e)

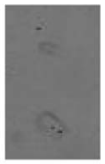

(f)
Fig. 6. (a), (b) Detailed subimages containing overlapping spots, (c), (d) vertical streaks, and (e), (f) artifacts, extracted from Fig. 5(f), (e), and (b), respectively.

two actual protein spots out of 57 and 26, respectively. In the case of the 2-D PAGE image in Fig. 5(f), the proposed technique misses 17 out of a total of 154 protein spots. These $F N$ cases are a side effect of median filtering, which may affect some regional intensity maxima.

Fig. 6 illustrates detailed subimages of the images in Fig. 5, so as to focus on the response of the proposed technique with respect to the presence of overlapping spot regions, streaks, and artifacts.

The detailed subimages in Fig. 6(a)-(b) depict complex regions with multiple overlapping spots. In both cases, it manages to separately detect each overlapping spot. This discriminative capability of the proposed technique is attributed to the size of the SE, which is smaller than the protein spot sizes.

Fig. 6(c) and (d) illustrates subimages containing multiple vertical streaks. Such streaks occur due to flawed calibration or insufficient SDS in electrophoresis buffer and pose difficulties for the consistent spot detection by biologists. The main challenge is the localization of protein spots within streaks. The proposed technique succeeds in detecting such spots. The $D S$ of the proposed technique in such cases can be attributed to the utilization of the disk-shaped SE, which tends to ignore the rectangular-shaped streaks.

Fig. 6(e) and (f) presents subimages containing artifacts. In both cases, the proposed technique correctly ignores all artifacts present. This can be attributed to the selectivity of the constraints imposed, which are parameterized so as to select salient intensity maxima.

The total execution time of the MATLAB implementation of the proposed technique for a 2-D PAGE image is approximately $15 \mathrm{~s}$.

The experimental results obtained from the proposed technique are compared to the results obtained by the application of Melanie software package on the same images. Fig. 7(a)-(c) illustrates the ground truth images, whereas Fig. 7(d)-(f) depicts the detection results obtained directly by the Melanie software package. It can be observed that much more actual protein spots are missed, whereas more artifacts are falsely detected as spots, 


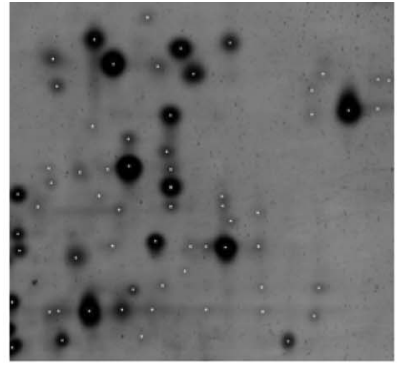

(a)

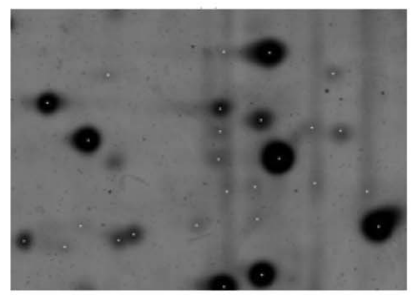

(b)

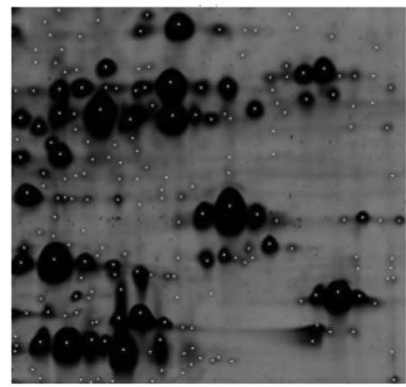

(c)

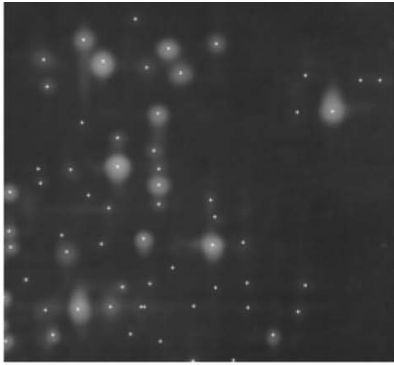

(d)

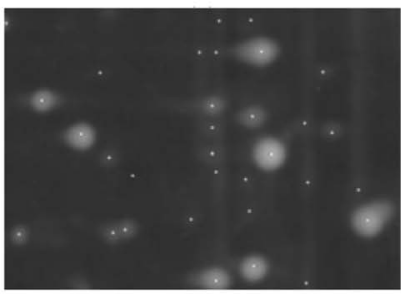

(e)

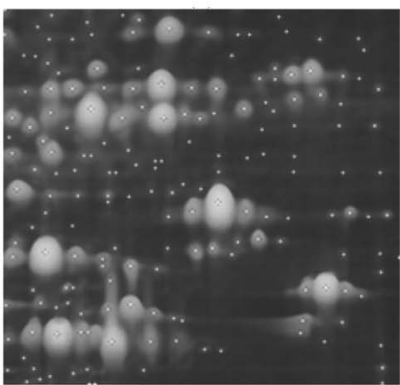

(f)
Fig. 7. (a)-(c) Ground truth images. (d)-(f) Detection results obtained by the Melanie software package.

TABLE II

INDICATIVE DETECTION RESULTS OBTAINED By MELANIE

\begin{tabular}{cccc}
\hline Image of Fig. 7 & $P V(\%)$ & $S P(\%)$ & $D S(\%)$ \\
\hline d & 90 & 43 & 90 \\
e & 90 & 35 & 89 \\
f & 75 & 25 & 85 \\
\hline
\end{tabular}

especially in the case of Fig. 7(f). Table II presents the $P V, S P$, and $D S$ obtained by the Melanie software package for each 2-D PAGE image in Fig. 7. The comparison of $P V, S P$, and $D S$ presented in Tables I and II leads to the conclusion that the Melanie software package is outperformed by the proposed technique in all image cases.

Table III presents the $P V, S P$, and $D S$ obtained by the proposed technique and Melanie, in a total of approximately 26000 protein spots appearing in a dataset of 13 2-D PAGE images. Fig. 8 provides a visualization of the results of Table III. It is evident that the proposed technique outperforms Melanie in terms of all performance criteria. In addition, analysis-ofvariance (ANOVA) has been performed so as to validate the statistical significance of the obtained results. ANOVA involves the so-called null hypothesis testing, where the null hypothesis is that the means among the compared populations are equal,
TABLE III

Overall Detection Results ObTained by the Proposed TECHNIQUE AND MELANIE

\begin{tabular}{lcccc}
\hline & \multicolumn{2}{c}{ Proposed Technique } & \multicolumn{2}{c}{ Melanie } \\
\hline & mean & $\begin{array}{c}\text { standard } \\
\text { deviation }\end{array}$ & mean & $\begin{array}{c}\text { standard } \\
\text { deviation }\end{array}$ \\
\hline$P V(\%)$ & 88.2 & 4.2 & 73.6 & 17.4 \\
$S P(\%)$ & 81.6 & 5.3 & 33.2 & 13.5 \\
$D S(\%)$ & 87.3 & 6.2 & 77.4 & 12.6 \\
\hline
\end{tabular}

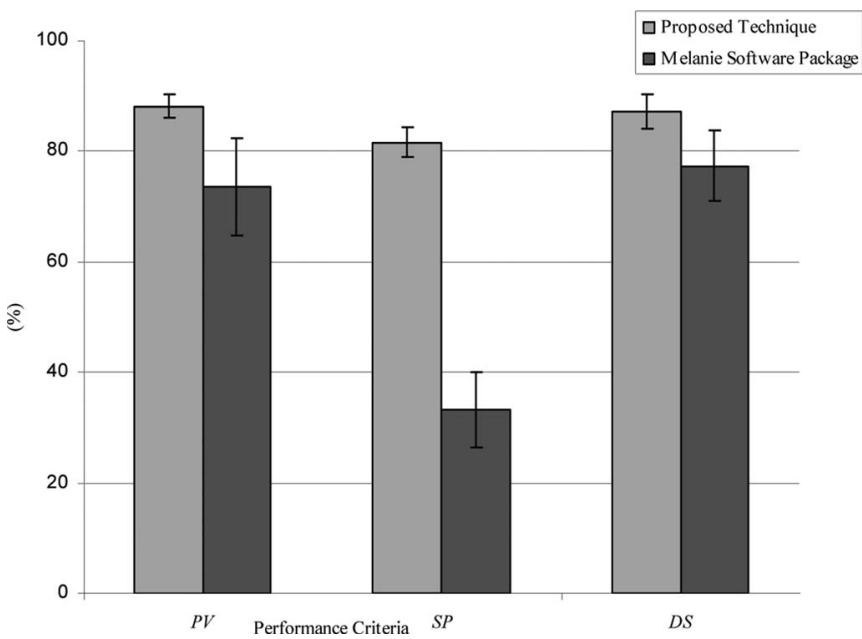

Fig. 8. Overall detection results in terms of $P V, S P$, and $D S$, obtained by the proposed technique and Melanie software.

under the assumption that these populations are normally distributed [27]. ANOVA yielded a $p$-value of less than 0.05 for all performance criteria, indicating that the performance difference of the proposed technique and Melanie is statistically significant.

The total execution time of the Melanie software package for a 2-D PAGE image is approximately $11 \mathrm{~s}$, which is slightly smaller than the time required by the MATLAB implementation of the proposed technique. However, it should be noted that this difference in execution time is not critical for the application. In addition, a $\mathrm{C}$ implementation of the proposed technique is expected to decrease the execution time.

\section{CONCLUSION}

In this paper, a novel computer-based technique is proposed for automated detection of protein spots in 2-D PAGE images. The dilation operator is used for the location of regional intensity maxima associated with protein spots. The SE is selected as disk shaped in accordance with the dominant roundish shape of most protein spots. The disk-shaped SE tends to ignore the rectangular-shaped streaks. In addition, SE radius $r$ is set considering that a certain radius value minimizes the detection of $F N$, whereas it allows the detection of local maxima associated with small spots even in cases where they overlap with larger spots in complex regions. The proposed technique imposes regional intensity constraints on the dilation-generated maxima so as to cope with the presence of noise and artifacts, such as 
cracks and dust particles. Furthermore, it is automated, efficient, and consistent with the time-consuming work of biologists.

Considering the experimental evaluation it can be concluded that the proposed technique:

1) achieves a $P V$ and a $D S$ which exceeds $90 \%$;

2) outperforms Melanie software package;

3) distinguishes multiple overlapping spots;

4) locates spots within streaks;

5) ignores artifacts; and

6) requires approximately $15 \mathrm{~s}$ to execute.

Future perspectives of this paper involve the segmentation of protein spots for the calculation of their mass and the development of an integrated 2-D PAGE image analysis system for the identification of protein spots.

\section{ACKNOWLEDGMENT}

The authors would like to thank the expert biologists A. Vlahou and S. Garbis of the Biomedical Research Foundation of the Academy of Athens for the provision of ground truth of 2-D PAGE images.

\section{REFERENCES}

[1] A. W. Dowsey, J. D. Michael, and G. Yang, "The role of bioinformatics in two-dimensional gel electrophoresis," Proteomics, vol. 3, pp. 1567-1596, 2002.

[2] Y. Wu, P. Lemkin, and K. Upton, "A fast spot segmentation algorithm for two-dimensional gel electrophoresis analysis," Electrophoresis, vol. 14, pp. 1351-1356, 1993.

[3] L. Vincent and P. Soille, "Watersheds in digital spaces: An efficient algorithm based on immersion simulations," IEEE Trans. Pattern Anal. Mach. Intell., vol. 13, no. 6, pp. 583-598, Jun. 1991.

[4] P. Cutler, G. Heald, I. R. White, and J. Ruan, "A novel approach to spot detection for two-dimensional gel electrophoresis images using pixel value collection," Proteomics, vol. 3, pp. 392-401, 2003.

[5] G. W. Horgan and C. A. Glasbey, "Uses of digital image analysis in electrophoresis," Electrophoresis, vol. 16, pp. 298-305, 1995.

[6] Y. Kim, J. Kim, Y. Won, and Y. In, "Segmentation of protein spots in 2-D gel electrophoresis images with watershed using hierarchical threshold," LNCS, vol. 2869, pp. 389-396, 2003.

[7] M. H. Tsai, H. H. Hsu, and C. C. Cheng, "Watershed-based protein spot detection in 2DGE images," in Proc. Int. Comput. Symp., 2007, pp. 1334 1338.

[8] E. R. Dougherty and R. A. Lotufo, Hands-On Morphological Image Processing. Bellingham, WA: SPIEPress, 2003.

[9] F. Y. Shih and Y. T. Wu, "Decomposition of arbitrary gray-scale morphological structuring elements," Pattern Recognit., vol. 38, pp. 2323-2332, 2005.

[10] J. Serra, Image Analysis and Mathematical Morphology. London: Academic, 1982.

[11] R. M. Haralick, S. R. Sternberg, and X. Zhuang, "Image analysis using mathematical morphology," IEEE Trans. Pattern Anal. Mach. Intell., vol. 9, no. 4, pp. 532-550, Jul. 1987.

[12] L. Vincent, "Morphological grayscale reconstruction in image analysis: Applications and efficient algorithms," IEEE Trans. Image Process., vol. 2, no. 2, pp. 176-201, Apr. 1993.

[13] H. J. A. M. Heijmans, Morphological Image Operators. Boston, MA: Academic, 1994.

[14] P. Soille, Morphological Image Analysis-Principles and Applications. Berlin, Germany: Springer, 1999.

[15] B. Bödvarsson, S. Klim, M. Mørkebjerg, S. Mortensen, C. H. Yoon, J. Chen, J. R. Maclaren, P. K. Luther, J. M. Squire, P. J. Bones, and R. P. Millane, "A morphological image processing method for locating myosin filaments in muscle electron micrographs," Image Vis. Comput., vol. 26, pp. 1073-1080, 2008.

[16] T. Li, S. Wang, and N. Zhao, "Gray-scale edge detection for gastric tumor pathologic cell images by morphological analysis," Comput. Biol. Med., vol. 39, pp. 947-952, 2009.
[17] D. Welfer, J. Scharcanski, C. M. Kitamura, M. M. Dal Pizzol, L. W. B. Ludwig, and D. R. Marinho, "Segmentation of the optic disk in color eye fundus images using an adaptive morphological approach," Comput. Biol. Med., vol. 40, pp. 124-137, 2010.

[18] M. M. Skolnick, "Application of morphological transformations to the analysis of two-dimensional electrophoresis gels of biological materials," Comput. Vis. Graph Image Process., vol. 35, pp. 306-322, 1986.

[19] P. Peer and L. G. Corzo, "Local pixel value collection algorithm for spot segmentation in two-dimensional gel electrophoresis research," Comput. Funct. Genomics, vol. 2007, Article ID 89596, 2007.

[20] Z. Lacroix, "Biological data integration: Wrapping data and tools," IEEE Trans. Inf. Technol. Biomed., vol. 6, no. 2, pp. 123-128, Jun. 2002.

[21] J.-Y. Bansard, D. R. Schuhman, G. Cameron, D. Clark, E. Mulligen, F. Beltrame, E. D. H. Barbolla, F. M. Sanchez, L. Milanesi, I. Tollis, J. V. Lei, and J.-L. Coatrieux, "Medical informatics and bioinformatics: A bibliometric study," IEEE Trans. Inf. Technol. Biomed., vol. 11, no. 3, pp. 237-243, May 2007.

[22] M. Savelonas, D. Maroulis, and E. Mylona, "Segmentation of twodimensional gel electrophoresis images containing overlapping spots," in Proc. IEEE Int. Conf. Inf. Technol. Appl. Biomed. Larnaca, Cyprus, Nov. 5-7, 2009, pp. 1-4.

[23] M. Coster and J.-L. Chermant, Précis d'analyse d'images. Paris, France: Presses du CNRS, 1989.

[24] F. G. Huang and G. Yang, "The application of soft morphology in image edge detection," J. Image Graph. China, vol. 5, pp. 284-288, 2000.

[25] D. T. Lin, "Autonomous sub-image matching for two-dimensional electrophoresis gels using MaxRST algorithm," Image Vis. Comput., vol. 28 , no. 8, pp. 1267-1279, Aug. 2010.

[26] C. Guda, E. Fahy, and S. Subramaniam, "MITOPRED: A genome-scale method for prediction of nucleus-encoded mitochondrial proteins," Bioinformatics, vol. 20, pp. 1785-1794, 2004

[27] S. Theodoridis and K. Koutroumbas, Pattern Recognition, 4th ed. San Diego, CA: Academic, 2008.

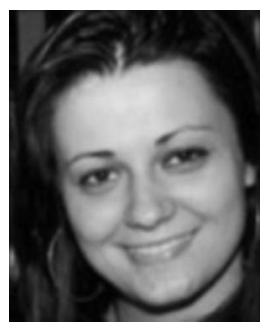

Eleftheria A. Mylona received the B.Sc. degree in applied mathematical and physical sciences from the National Technical University of Athens, Athens, Greece, in 2006 and the M.Sc. degree in physics from the University of Edinburgh, Edinburgh, Scotland, in 2007. She is currently working toward the Ph.D. degree in biomedical image analysis at the University of Athens, Athens, Greece.

She has coauthored four research articles on biomedical image analysis. Her research interests include image analysis, segmentation, and

bioinformatics

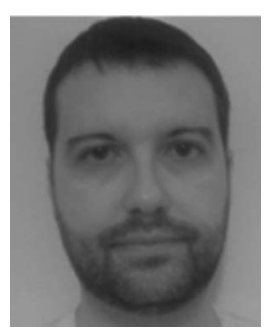

Michalis A. Savelonas (M'08) received the B.Sc. degree in physics in 1998, the M.Sc. degree (Hons.) in cybernetics in 2001, and the Ph.D. degree (Hons.) in the area of image analysis in 2008, all from the University of Athens, Athens, Greece. For the Ph.D. research, he received a scholarship by the Greek General Secretariat of Research and Technology (25\%) and the European Social Fund (75\%).

From 2002 to 2004, he was with software industry for several projects involving the development of real-time systems. He has coauthored more than 30 scientific articles in peer-reviewed international journals, conference proceedings, and book chapters, whereas he has been actively involved in seven European and National R\&D projects. He is a reviewer in various international journals including Pattern Recognition and Signal Processing. Among positions in various academic institutions, which include University of Athens, Hellenic Air Force Academy, and Technological and Educational Institute of Lamia, he is a Research Fellow in the Department of Informatics and Telecommunications, University of Athens, Greece, as well as in Information Technology Institute, Center for Technological Research of Central Greece, Lamia, Greece. His research interests include image analysis, segmentation, pattern recognition, medical applications, bioinformatics, and watermarking. 


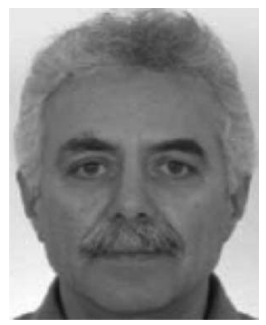

Dimitris Maroulis (M’02) received the B.Sc. degree in physics, the M.Sc. degree in radioelectricity and in cybernetics with honors, and the Ph.D. degree in computer science with honors, all from the University of Athens, Athens, Greece.

He served as a Research Fellow for three years at the Space Research Department (DESPA) of Meudon Observatory, Paris, France, and afterward he collaborated for more than ten years with the same department. He is currently a Professor in the Department of Informatics and Telecommunications of the University of Athens and leader of the Real Time Systems and Image Analysis Lab. He has more than 20 years of experience in the areas of data acquisition and real-time systems, and more than 15 years of experience in the area of image/signal analysis and processing. He has also been collaborating with many Greek and European hospitals and health centers for more than 12 years in the field of biomedical informatics. He has been actively involved in more than 12 European and National R\&D projects and has been the project leader of 5 of them, all in the areas of image/signal analysis and real-time systems. He has published more than 130 research papers and book chapters, and there are currently more than 650 citations that refer to his published work. He is a reviewer in more than 12 international journals including Pattern Recognition, IEEE TRANSACTIONS ON INFORMATION TECHNOLOGY IN BIOMEDICINE, and IEEE TRANSACTIONS ON MEDICAL IMAGING. His research interests include data acquisition and real-time systems, pattern recognition, image/signal processing and analysis, with applications on biomedical systems and bioinformatics.

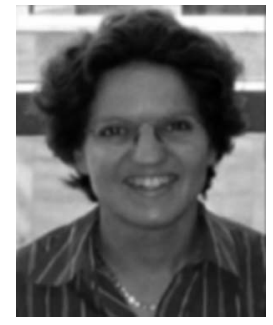

Sophia Kossida received the B.Sc. degree in biology from the University of Crete, Crete, Greece, in 1995. She was awarded the IKY Governmental Scholarship for three successive years given to the student with the best grades by the University of Crete. She received the free-mover Erasmus scholarship and spent a year in Trinity College of Dublin, in Ireland within the Genetics Department, Bioinformatics Unit, where she focused on the genome of Saccharomyces cerevisiae. She received the full medical research council (MRC) scholarship to carry out the D.Phil. from Oxford University, Merton College, U.K., in 1998.

She did extensive research on sequence analysis of viral genomes (DNA viruses and retroviruses) and deciphered their evolutionary history. She was a Postdoctoral Researcher at Harvard University, Cambridge, MA, in the Molecular and Cellular Biology Department within the FlyBase group, holding an NIH fellowship. She was a Senior Scientist within the Target Discovery Group of Lion Bioscience Research, Inc. (LBRI), Cambridge, MA, where she was involved in the human genome mining project. She was the coordinator between LBRI and the Bayer Diabetes group in the U.S. and in Germany as well as the Scientist in charge for the patent applications. She moved over to Toulouse, in France, where she became the Director of Bioinformatics of Endocube, a start up company focusing $n$ endothelial cells. In parallel, she was an Associate Professor of Bioinformatics at the University of Paul Sebatier, Toulouse, France. She joined Novartis in Switzerland in 2002 as a Laboratory Head within the Functional Genomics Group. She worked on a few different sequence analysis projects with main focus on proteases. She joined the Biomedical Research Foundation of the Academy of Athens (BRF) in July 2004 as tenure track research Bioinformatician, Center of Basic Research II, Biotechnology Division. She holds 11 published patent applications. Her team within BRF was appointed the National Contact Point for Bioinformatics for the EMBnet. 